\title{
Redes Neurais Convolucionais para Classificação da COVID-19 em Imagens de Raio-x de Tórax
}

\author{
$1^{\text {st }}$ Ricardo de Sousa Farias $\quad 2^{\text {nd }}$ Kaique Rijkaard de Sousa Oliveira $3^{\text {rd }}$ Carlos Danilo Miranda Regis \\ Pós-Graduação em Engenharia Elétrica Pós-Graduação em Engenharia Elétrica Pós-Graduação em Engenharia Elétrica \\ Instituto Federal da Paraíba - IFPB Instituto Federal da Paraíba - IFPB \\ João Pessoa, Brasil \\ João Pessoa, Brasil \\ ricardo.farias@academico.ifpb.edu.br kaique.rijkaard@academico.ifpb.edu.br \\ Instituto Federal da Paraíba - IFPB \\ João Pessoa, Brasil \\ danilo.regis@ifpb.edu.br
}

\author{
$4^{\text {th }}$ Cecília de Moura Costa \\ Pós-Graduação em Medicina (Radiologia) \\ Universidade Federal do Rio de Janeiro - UFRJ \\ Rio de Janeiro, Brasil \\ ceciliamcost@gmail.com
}

\author{
$5^{\text {th }}$ José Manoel de Seixas \\ Laboratorio de Processamento de Sinais, COPPE/POLI \\ Universidade Federal do Rio de Janeiro - UFRJ \\ Rio de Janeiro, Brasil \\ seixas@1ps.ufrj.br
}

\begin{abstract}
Resumo-A COVID-19 tem causado pânico e transtornos pelo mundo, gerando altas taxas de internação em Unidades de Terapia Intensiva (UTI). Os infectados por COVID-19, geralmente, apresentam sintomas que acometem o sistema respiratório. A análise de imagens de raio-x de tórax têm auxiliado o entendimento do quadro clínico dos pacientes com COVID-19, possibilitando uma melhor perspectiva dos impactos causados no pulmão. Este trabalho aborda a classificação de imagens de raio-x em três classes distintas: COVID-19, Normal e Pneumonia Viral, utilizando oito diferentes modelos convolucionais de aprendizado profundo, sendo eles a VGG16, VGG19, InceptionV3, ResNet50, InceptionResNetV2, Xception, DenseNet201 e NASNetLarge. Foi utilizado as medidas de desempenho de acurácia, sensibilidade, especificidade, precisão, pontuação F1 e AUC (Área sob a curva ROC). Na classificação binária de COVID-19 e Normal, o modelo Xception se destacou na maioria da medidas de desempenho com valores em torno de $99 \%$. Na classificação binária entre COVID-19 e Pneumonia Viral, o modelo DenseNet201 alcançou os melhores resultados na maioria das medidas de desempenho em torno de $98 \%$. Na classificação multiclasse a DenseNet201 alcançou os melhores resultados em quatro das seis medidas de desempenho com valores entre $94 \%$ e $96 \%$.

Palavras-chave-COVID-19, Raio-x, Redes neurais convolucionais, Classificação de imagens
\end{abstract}

\section{INTRODUÇÃO}

No final de 2019, na cidade de Wuhan, localizada na China, iniciou um surto viral causado pelo vírus SARSCoV-2, também conhecido como novo coronavírus (COVID19) [1], [2]. Em 11 de março de 2020, a Organização Mundial de Saúde (OMS) declarou o surto de SARS-CoV-2 como uma pandemia, devido ao crescente número de casos fora da China [3]. Embora declarada pandemia pela OMS, em 11 de março de 2020, no Brasil, o primeiro paciente foi diagnosticado em fevereiro do mesmo ano [4]. Diversos campos como social, econômico e político foram atingidos pela pandemia o que causou diversos problemas em todo o mundo. No mundo, o numero de infectados já passa dos 205 milhões de pessoas e mais de 4 milhões de mortes foram registradas.
A busca por agilidade na identificação da COVID-19 têm impulsionado o desenvolvimento de formas alternativas para diagnóstico, como modelos de aprendizado profundo (DL Deep learning).

Aplicações de DL tem sido utilizados em tarefas de análise de imagens médicas, como classificação , segmentação e registro [5]. Os bons resultados alcançados por esses modelos tem ganhado cada vez mais espaço nas diversas áreas da saúde, como na identificação de Alzheimer e tumor cerebral por meio da ressonância magnética [6] [7]; detecção de câncer de mama em imagens de mamografia [8]; detecção da tuberculose, pneumonia e COVID-19 através de imagens de raio-x do tórax (CXR - Chest X-ray) [9] [10]; câncer de pulmão com imagens de tomografias computadorizadas [11]; dentre outras aplicações.

As radiografias de tórax são comumente requeridas para os pacientes com sintomas pulmonares agudos e críticos por possuírem menor custo e baixa exposição a radiação [12]. Um dos primeiros trabalhos com foco na detecção da COVID-19, utilizando imagens de raio-x do tórax, foi desenvolvido por Hemdan et al. [13]. O trabalho propôs um modelo chamado de COVIDX-Net, no qual abordou diferentes modelos neurais convolucionais, entre elas, as que se destacaram foram a VGG19 e DenseNet201, que obtiveram resultados acima de $90 \%$ de acurácia.

Outra pesquisa desenvolvida com imagens de raios-X do tórax foi a de Nour e Cömert [14] que projetaram um modelo de Rede Neural Convolucional (CNN - Convolutional Neural Network) com 5 camadas para extrair características discriminativas, essas características que foram aplicadas como entradas para algoritmos de aprendizado de máquina. O SVM obteve os melhores resultados, acurácia de 98,97\%, sensibilidade de $89,39 \%$ e especificidade de $99,75 \%$.

O trabalho de [10] propôs um modelo de Rede Neural Convolucional empilhada e integrada no qual faz uso de outras arquiteturas de CNN pré-treinadas, foram aplicadas 
na detecção de 3 classes, Normal, Pneumonia e COVID-19, atingindo uma acurácia de 99,08\%. Na classificação binária, COVID-19 e não COVID-19 obtiveram uma acurácia de $99 \%$.

No estudo de [15], utilizaram quatro modelos de CNNs, a DenseNet121, ResNet50, VGG16, e VGG19 para o diagnóstico de imagens de raios-X como COVID-19 ou Normal. No estudo constatou-se que a VGG16 e VGG19 superaram os outros dois de CNNs e alcançaram uma precisão de 99,3\%.

Neste contexto, o presente trabalho tem como objetivo classificar, a partir da análise de imagens de raio-x do tórax, a COVID-19, Pneumonia Viral e o estado Normal (saudável) utilizando diferentes redes neurais convolucionais.

\section{REDEs Neurais CONVOluCiOnAis}

O aprendizado de máquina (ML - Machine Learning) faz parte da grande área da Inteligência Artificial, dentro dele têm-se o aprendizado profundo (DL - Deep Learning) com o propósito de automatizar a identificação de padrões significativos nos dados, e desse modo, resolver problemas por meio de algoritmos. As Redes Neurais Convolucionais se enquadram nas técnicas de DL e são amplamente utilizadas em tarefas de reconhecimento de padrões de imagens, por apresentarem como característica a aptidão superior de aprendizagem de recursos [16].

As CNNs têm sido constantemente utilizadas em aplicações que envolvem imagens médicas. A utilização das CNNs têm favorecido a uma melhor compreensão dessas imagens em um menor custo de tempo. As CNNs, na análise de imagens médicas, normalmente, realizam tarefas de classificação, detecção e segmentação [17].

Uma CNN é basicamente estruturada em três camadas: camada de entrada, camada oculta e camada de saída, semelhante às Redes Neurais Artificiais (RNA) convencionais. A diferença entre elas está na camada oculta, onde na CNN é normalmente subdividida em três camadas: camada convolucional, camada de subamostragem (pooling) e camada totalmente conectada [17].

A camada de convolução utiliza-se de filtros para realizar a extração de recursos da entrada, e a partir da extração, obtémse características de alto nível. Os filtros percorrem todos os dados da imagem em largura, altura e profundidade, aplicando a operação de convolução. Conforme o treinamento da rede, ocorre um ajuste nos filtros, que torna possível identificar características que os lotes de entradas possuem em comum como cores, bordas, arestas, entre outras. À medida que as entradas vão sendo passadas para a rede, os filtros conseguem aprender características mais complexas [18]. Isso resulta no reconhecimento de mesmas características ou até mesmo o objeto em várias imagens em locais diferentes [19].

Após a camada de convolução é utilizado a camada pooling. A camada de pooling normalmente é utilizada para construir o mapa de recursos agrupando as informações advindas das camadas anteriores. Esta camada tem como objetivo reduzir os dados de entrada. Com a redução dos dados de entrada, também há a redução do custo computacional evitando assim o overfitting. As camadas totalmente conectadas estão localizadas no final da rede. Após as características serem extraídas, as camadas totalmente conectadas utilizam essas características para produzir a resposta de classificação da rede.

$\mathrm{Na}$ construção de modelos de CNN, as funções de ativação são elementos fundamentais, pois têm como função decidir se um neurônio deve ser ativado ou não conforme a relevância da informação [20].

A função de ativação ReLU gera valores entre 0 e infinito (Eq. 1). Quando os valores são negativos o retorno é 0 , quando o valor é positivo o retorno é o próprio valor [21]. Dessa forma, para ativar o neurônio é preciso que o valor seja maior que zero, isso o faz ser esparso e eficiente [20].

$$
\operatorname{ReLu}(x)=\max \{0, x\}
$$

A função de ativação Softmax é muito utilizada em problemas de classificação e em um grande número de tarefas, é aplicada na camada de saída do classificador para determinar a qual classe pertence cada entrada. É realizada uma transformação da saída para valores entre 0 e 1 para cada classe, sendo dividido pela soma das saídas, o que resulta na probabilidade da informação de entrada estar em uma determinada classe [20]. A função é definida conforme a Eq. 2.

$$
\sigma(Z)_{j}=\frac{e^{z_{j}}}{\sum_{k=1}^{K} e^{z_{k}}} \quad \text { para } j=1, \ldots, K
$$

Grande parte do sucesso, nas aplicações envolvendo as técnicas de DL, principalmente as $\mathrm{CNNs}$, está relacionado a utilização de grandes conjuntos de treinamento. O processamento grandes volumes de dados requer máquinas com grande capacidade computacional, além de um longo tempo para o treinamento. Entretanto, esses recursos podem não estar disponíveis, o que torna o processo de treinamento inviável [22].

A aprendizagem por transferência é um método robusto de aprendizagem profunda que reaproveita o conhecimento aprendido em uma outra tarefa para executar uma dada tarefa [23]. No decorrer da execução do aprendizagem de transferência, todas as camadas são treinadas, não treinando apenas a camada totalmente conectada. Uma maneira de ganhar desempenho do modelo é treinar apenas as camadas CNN completa ou parcialmente, permitindo que os pesos se ajustem a partir dos recursos do conjunto de dados pré-treinados do ImageNet para os recursos especializados do conjunto de dados de imagens de raio-X [24].

Neste trabalho, os modelos utilizados para realizar as classificações foram os modelos pré-treinados no conjunto de dados do ImageNet: VGG16, VGG19, InceptionV3, DenseNet201, ResNet50, Xception, InceptionResNetV2 e NASNetLarge. É aplicado o conceito de aprendizagem por transferência. Um ajuste fino deste aprendizado acontece nas camadas totalmente conectadas. Internamente são ativadas com função ReLu nas camadas intermediaria e softmax na camada totalmente conectada, extraíram recursos que permitiram realizar a classificação. 


\section{A. VGG16 e VGG19}

A VGG é uma arquitetura que utiliza camadas convolucionais de kernel $3 \times 3$ empilhadas com profundidade crescente. Após cada pilha de convolução é aplicado o pooling máximo [25]. As VGGs possuem três camadas totalmente conectadas (Full conected) as duas primeiras com 4096 neurônios e a ultima realiza a classificação, com a função softmax, que distribui probabilidades que caracterizam as classes. São aplicadas em todas as camadas ocultas a função de ativação $\mathrm{ReLu}$ [26]. A regularização dropout ocorre nas camadas totalmente conectadas [27]. A diferença entre a VGG16 e VGG19 diz respeito ao número de camadas, pois a VGG19 possui três camadas convolucionais adicionais, resultando em um maior de parâmetros. Esses modelos como os outros que foram utilizados neste trabalho estão disponíveis pré-treinados na biblioteca do Keras.

\section{B. InceptionV3}

A InceptionV3 é uma arquitetura de 48 camadas de profundidade. O foco é em problemas de classificação de imagens [26]. A sua arquitetura possui diversos blocos de construção regulares e irregulares com separações de convoluções, média, camadas de pooling máximo, camadas concatenadas, dropouts e camadas totalmente conectadas [28]. Essa arquitetura possui menor custo computacional, menor número de parâmetros, quando comparada com as VGGs [29].

\section{DenseNet201}

A DenseNet201 é uma CNN que possui um padrão de conectividade simples, onde todas as camadas são conectadas diretamente entre si com mapas de características de tamanhos correspondentes, garantindo entre as camadas o fluxo máximo de informações. Cada camada retém entradas adicionais das camadas antecedentes e transita seus respectivos mapas de características para camadas subsequentes. As características são concatenadas antes de serem enviadas para uma camada. Esta rede têm a vantagem de atenuar o problema do esvanecimento do gradiente, além de fortalecer a propagação de características, incentivar o reuso de características e, assim diminuir a quantidade de parâmetros [30].

\section{D. $\operatorname{ResNet50}$}

A ResNet50 é uma rede do tipo residual de 50 camadas. Conforme [26] a ResNet50 possui uma estrategia de "atalhos", a ideia por trás dos atalhos é pular pares de grupos de camadas convolucionais, a rede conforme vai ficando mais profunda, não morrerá de esvanecimento de gradientes. Outra característica importante desta rede é seu grande foco em normalização de lotes. Esta rede detêm camadas que se ajustam a um mapeamento residual explicitamente, ao invés de aguardar que as camadas empilhadas se regulem diretamente a um desejado mapeamento subjacente.

\section{E. Xception}

É uma CNN inspirada na Inception, onde os módulos Inception foram substituídos por convoluções separáveis em profundidade. A Xception superou a InceptionV3 no conjunto de dados da ImageNet para qual a InceptionV3 foi projetada. A Xception tem o mesmo número de parâmetros que a Inception, entretanto, o ganho da Xception se dá pelo uso mais eficiente dos parâmetros do modelo [31].

A base de extração de características da rede Xception é composta por 36 camadas convolucionais. As camadas possuem uma estrutura com 14 módulos, sendo que todos tem em suas proximidades as conexões residuais lineares, com exceção do primeiro e último módulo [31].

\section{F. InceptionResNetV2}

A InceptionResNetV2 é uma CNN que combina as propriedades da Inception e da conexão residual do ResNet, contendo 162 camadas de rede profundas [32]. No bloco Inception-Resnet, filtros convolucionais de vários tamanhos são combinados com conexões residuais, essas conexões reduz o custo de tempo no treinamento e evita o problema de degradação causado por estruturas profundas [33]. As conexões residuais mais profundas permitem atalhos no modelo, o qual pode permitir modelos mais profundos, levando a um melhor desempenho [34].

\section{G. NASNetLarge}

A NASNet é uma família de modelos que foram projetados automaticamente, aprendendo as arquiteturas de modelos diretamente no conjunto de dados de interesse. A NASNetLarge é uma CNN que foi treinada em mais de um milhão de imagens do banco de dados ImageNet. Esta rede pode classificar mais de 100 categorias. A rede aprendeu representações de recursos avançados para uma ampla gama de imagens. Mas, é exigido um alto poder computacional [35].

\section{iII. Materiais e Metodologia}

Para ser realizada a identificação de quais pacientes estão com COVID-19, foi realizada a classificação utilizando imagens de raio-x do tórax (COVID-19 Radiography Database), onde as imagens são pré-processadas e redimensionadas. Para o treinamento foram utilizados diferentes modelos e as classes da base de dados: COVID-19, Pneumonia Viral e Normal. Na Figura 1 é apresentado o fluxograma com as etapas realizadas.

Com o propósito de realizar a identificação da COVID-19, por meio da análise de imagens de raio-x do tórax, alguns passos foram realizados como é ilustrado na Figura 1. Foi selecionada a base de imagens de raio-x do tórax contendo imagens de pulmões em estado Normal, com Pneumonia Viral e COVID-19. Em seguida, as imagens se tornaram dados de entrada para o treinamento de modelos de classificação binária e multiclasse. Foram realizadas classificações utilizando três classes, definidas como: COVID-19, Pneumonia Viral e Normal. Oito modelos pré-treinados realizaram as classificações e foram avaliados segundo medidas de desempenho como acurácia, sensibilidade, especificidade, precisão, pontuação $\mathrm{F} 1$, 


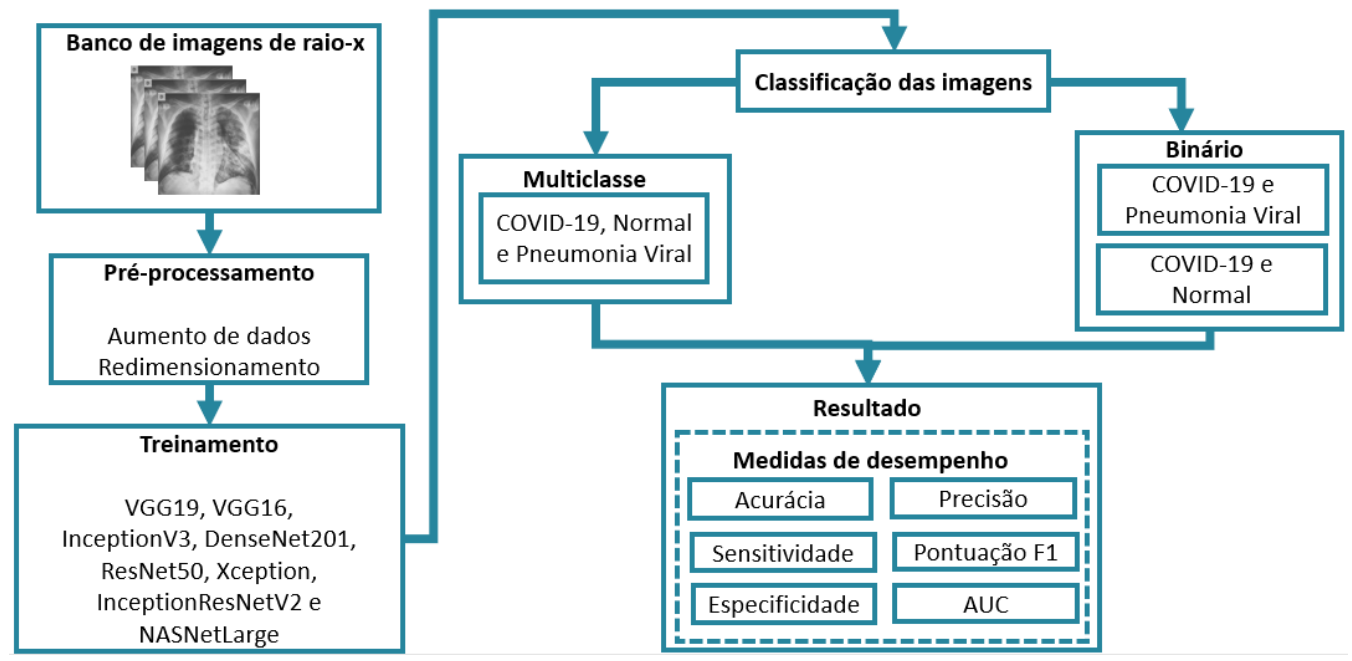

Figura 1. Metologia proposta para a classificação de COVID.

AUC e plotado as matrizes de confusão dos melhores modelos. Os passos se encontram mais detalhados nas subseções deste capitulo.

\section{A. Base de Dados}

A base de dados utilizada neste trabalho é a COVID19 Radiography Database [36]. Este banco de dados é de domínio público e está hospedado na plataforma Kaggle. O banco usado contêm imagens de raio-x do tórax, com vistas frontal, e é dividido em quatro classes: COVID-19, Opacidades Pulmonares, Pneumonia Viral e Normal. Entretanto, este trabalho tem como objetivo a classificação das classes COVID-19, Pneumonia Viral e Normal. O banco quando foi baixado e utilizado era composto por 1200 imagens de raio$\mathrm{x}$ de COVID-19, 1345 imagens de Pneumonia Viral e 1341 imagens normais. Recentemente foi realizada uma atualização no banco que contém mais imagens sendo 3616 imagens de COVID-19, 1345 de Pneumonia Viral e 10192 de Normal, mas essas novas imagens não foram usadas nesse trabalho.

\section{B. Pré-Processamento dos Dados}

Com o intuito de minimizar os possíveis erros na identificação dos objetivos do trabalho, bem como diminuir o esforço computacional necessário na classificação das imagens. As imagens foram redimensionadas de $256 \times 256$ para $150 \times 150$ por conta do custo computacional, normalizadas e aplicado o data augmentation com a rotação de 15 graus de modo que a rotação não prejudique as informações de ambos lado do pulmão. Além disso,o modo de preenchimento vizinho mais próximo também foi utilizado. $\mathrm{O}$ aumento de dados resulta na geração de novas imagens para que o modelo possa generalizar.

\section{Classificação da COVID-19}

Para realizar a identificação da infecção por COVID-19, foram utilizados diferentes modelos de aprendizado profundo, de modo a detectar com pacientes com o quadro Normal ou infectados por COVID-19, além de considerar também casos de Pneumonia, pois esta pode ser confundida com a COVID19, devido aos danos pulmonares semelhantes que pode causar.

Para o treinamento, foram utilizadas redes pré-treinadas (Seção II) e para cada uma delas foi aplicado um ajuste fino, para realizar a transferência do aprendizado. As configurações de treinamento foram: 50 épocas de treinamento com batchsize de 32 e taxa de aprendizado de 0,0001. A base de dados foi dividida, com $67 \%$ das imagens utilizadas para treinamento e $33 \%$ para teste. As redes neurais foram executadas separadamente para cada tipo de classificação (Tabela I).

Tabela I

DIVISÃO DOS TESTE REALIZADOS ENTRE CLASSIFICAÇÃO BINARIA E MULTICLASSE.

\begin{tabular}{|c|c|}
\hline Teste & Classificação \\
\hline 1 & COVID-19 e Normal \\
2 & COVID-19 e Pneumonia Viral \\
3 & COVID-19, Pneumonia Viral e Normal \\
\hline
\end{tabular}

\section{Medidas de desempenho}

Para medir o desempenho dos classificadores propostos, foram utilizados diferentes indicadores. As medidas de desempenho utilizadas neste trabalho foram: acurácia, sensibilidade, especificidade, precisão, pontuação F1 e Área sob a curva ROC (AUC - Area Under the ROC Curve), considerando-se também a matriz de confusão. Como o objetivo é a triagem de pacientes com infecção por COVID-19, ênfase maior é dada à sensibilidade e especificidade, são as duas principais medidas quando se trata de imagens medicas, são medidas precisas e científicas para mensurar a classificação dessas imagens para triagem médicas [37]. São consideradas importantes em imagens clinicas para confirmar ou excluir doenças durante a triagem [38].

\section{Resultados}

Nesta seção são apresentados os resultados alcançados por meio dos modelos pré-treinados nas classificações binárias e 
multiclasses (Tabela I) para a identificação de COVID-19. As classificações realizadas foram comparadas para verificar a eficiência dos modelos em imagens de raio-X, e avaliadas pelas as medidas de desempenho apresentadas na Seção III-D.

\section{A. Classificação COVID-19 e Normal}

Para a classificação COVID-19 e normal observa-se que o desempenho do modelo Xception obteve valores melhores aos demais nas medidas de desempenho com resultados em cerca de $99 \%$, com exceção da pontuação AUC que a Xception obteve $98,33 \%$, enquanto que a DenseNet201 atingiu o valor de 99,50\%, como apresentado na Tabela II. Percebe-se que os valores obtidos pela maioria dos modelos ficaram próximos.

Os modelos que se destacaram na sensibilidade foram a Xception, Inceptionv3 e DenseNet201 com valores em acima de $99 \%$, ao mesmo tempo que na especificidade todos os modelos atingiram resultados por volta de 99\%. Portanto, entende-se que os modelos Xception, Inceptionv3 e DenseNet201 estão mais equilibrados para a triagem, e conseguem ter resultados satisfatórios para distinguir um pulmão saudável de um acometido pela COVID-19.

Na Fig. 2, é exibida a matriz de confusão para o modelo Xception. Observa-se o bom desempemho nessa classificação, haja vista que 443 imagens foram classificadas corretamente como Normal e 395 como COVID-19, sendo que apenas 3 imagens foram classificadas erroneamente.

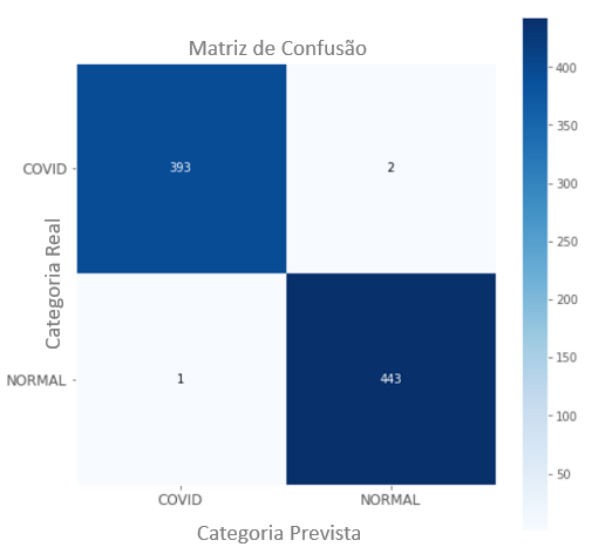

Figura 2. Matriz de confusão Xception de COVID-19 e Normal

\section{B. Classificação COVID-19 e Pneumonia Viral}

Verifica-se que o modelo DenseNet201 alcançou resultados superiores na maioria das medidas de desempenho, em torno de $98 \%$, contudo a NASNetLarge atingiu uma sensibilidade maior de $98,25 \%$ e a VGG19 obteve uma melhor especificidade de $100 \%$, como ilustrado na Tabela III. Por outro lado, a DenseNet201 foi o modelo que conseguiu o segundo melhor resultado na sensibilidade com $97,75 \%$, bem como na especificidade com 99,32\%.

Para essa classificação, observa-se uma flutuação maior nos resultados obtidos, quando os comparamos com o resultado anterior. Uma vez que as características da Pneumonia Viral são similares ao da COVID-19, a discriminação correta das doenças é dificultada. Contudo, os modelos DenseNet201 e NASNetLarge se mostraram mais eficiente para classificações em triagens médicas.

A matriz de confusão do modelo DenseNet201 é apresentada na Fig. 3, devido ter apresentado os melhores resultados entre os modelos. Na classificação, 391 foram classificados corretamente como Pneumonia Viral e 437 como COVID-19, enquanto que o restante foram classificados de forma errônea entre falsos positivos e negativos.

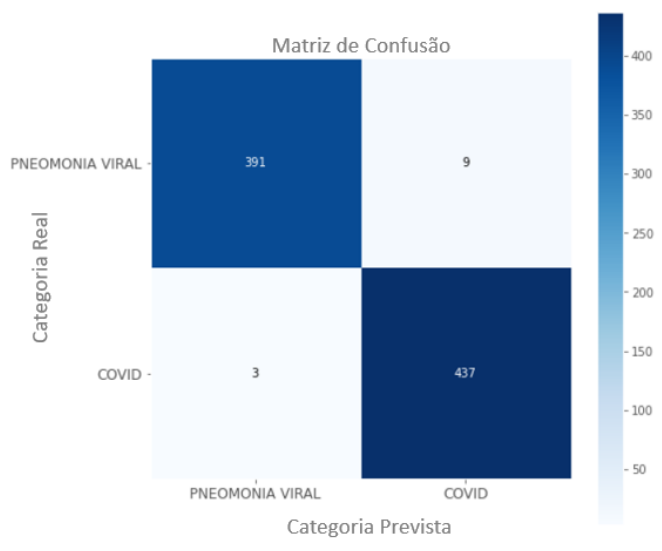

Figura 3. Matriz de confusão DenseNet201 de COVID-19 e Pneumonia Viral

\section{Resultados da classificação multiclasse: COVID-19, Pneu- monia Viral e Normal}

Esta perspectiva de classificação é relevante e sendo uma maneira para o diagnóstico final de um possível paciente infectado pela COVID-19. Os resultados da classificações apresentaram uma diminuição, quando comparado com as classificações anteriores. No entanto, os modelos conseguiram aprender e distinguir cada uma das classes utilizadas neste problema de classificação multiclasse.

A DenseNet201 destacou-se entre 4 das 6 diferentes medidas de desempenho. Se sobressaiu na acurácia, precisão, na pontuação F1 em cerca de $94 \%$ e AUC de 95,81\%. Enquanto que na sensibilidade, a NASNetLarge conseguiu um melhor resultado, 99,49\%, e a InceptionV3 uma maior especificidade, 99,78\%. Esses resultados são apresentados na Tabela IV.

Considerando a triagem médica, os modelos InceptionV3 e Xception alcançaram resultados mais balanceados para a sensibilidade e especificidade, com valores acima de $99 \%$.

A Fig. 4 ilustra a matriz de confusão da DenseNet201 na classificação multiclasse, no qual 384 foram classificados corretamente como Normal, 449 como COVID-19 e 356 como Pneumonia Viral, o restante foram classificados erroneamente entre as classes. Como é esperado a maior dificuldade na classificação foi diferenciar COVID-19 de pneumonia viral.

\section{Comparativo entre outros trabalhos que abordam a classificação de COVID-19}

Considerando os valores de acurácia de cada modelo no conjunto de imagens do ImageNet, com base no Keras Applications [39], esperava-se que os valores de acurácia fossem 
Tabela II

RESUlTAdos DAS CNN NA CLASSIFICAÇÃo BINÁRIA COVID-19 E NORMAL.

\begin{tabular}{|c|c|c|c|c|c|c|}
\hline & Acurácia & Sensibilidade & Especificidade & Precisão & Pontuação F1 & AUC \\
\hline VGG19 & $96,66 \%$ & $93,92 \%$ & $99,10 \%$ & $96,88 \%$ & $96,64 \%$ & $96,51 \%$ \\
VGG16 & $98,57 \%$ & $97,72 \%$ & $99,32 \%$ & $98,61 \%$ & $98,56 \%$ & $98,52 \%$ \\
InceptionV3 & $99,40 \%$ & $\mathbf{9 9 , 4 9 \%}$ & $99,32 \%$ & $99,40 \%$ & $99,40 \%$ & $99,40 \%$ \\
DenseNet201 & $99,52 \%$ & $\mathbf{9 9 , 2 4 \%}$ & $\mathbf{9 9 , 7 7 \%}$ & $99,54 \%$ & $99,52 \%$ & $\mathbf{9 9 , 5 0 \%}$ \\
ResNet50 & $89,39 \%$ & $77,72 \%$ & $\mathbf{9 9 , 7 7 \%}$ & $91,55 \%$ & $89,11 \%$ & $88,74 \%$ \\
Xception & $\mathbf{9 9 , 6 4 \%}$ & $\mathbf{9 9 , 4 9 \%}$ & $\mathbf{9 9 , 7 7 \%}$ & $\mathbf{9 9 , 6 5 \%}$ & $\mathbf{9 9 , 6 4 \%}$ & $98,33 \%$ \\
InceptionResNetV2 & $98,33 \%$ & $97,97 \%$ & $98,65 \%$ & $98,34 \%$ & $99,63 \%$ & $98,31 \%$ \\
NASNetLarge & $98,93 \%$ & $98,23 \%$ & $99,55 \%$ & $98,96 \%$ & $98,92 \%$ & $98,93 \%$ \\
\hline
\end{tabular}

Tabela III

RESUlTAdos dAS CNNS NA CLASSIFICAÇÃO BINARIA COVID-19 E PNEUMONIA VIRAL.

\begin{tabular}{|c|c|c|c|c|c|c|}
\hline & Acurácia & Sensibilidade & Especificidade & Precisão & Pontuação F1 & AUC \\
\hline VGG19 & $94,88 \%$ & $89,25 \%$ & $\mathbf{1 0 0 \%}$ & $95,55 \%$ & $94,83 \%$ & $94,62 \%$ \\
VGG16 & $96,90 \%$ & $95,50 \%$ & $98,18 \%$ & $96,97 \%$ & $96,89 \%$ & $96,84 \%$ \\
InceptionV3 & $97,50 \%$ & $96,00 \%$ & $98,86 \%$ & $97,58 \%$ & $97,49 \%$ & $97,43 \%$ \\
DenseNet201 & $\mathbf{9 8 , 5 7 \%}$ & $97,75 \%$ & $99,32 \%$ & $\mathbf{9 8 , 6 1 \%}$ & $\mathbf{9 8 , 5 7 \%}$ & $\mathbf{9 8 , 5 3 \%}$ \\
ResNet50 & $85,48 \%$ & $76,25 \%$ & $93,86 \%$ & $86,58 \%$ & $85,23 \%$ & $85,05 \%$ \\
Xception & $97,50 \%$ & $96,75 \%$ & $98,18 \%$ & $97,53 \%$ & $97,49 \%$ & $97,46 \%$ \\
InceptionResNetV2 & $96,90 \%$ & $96,50 \%$ & $97,27 \%$ & $96,91 \%$ & $96,90 \%$ & $96,88 \%$ \\
NASNetLarge & $98,45 \%$ & $\mathbf{9 8 , 2 5 \%}$ & $98,64 \%$ & $98,45 \%$ & $98,45 \%$ & $98,44 \%$ \\
\hline
\end{tabular}

Tabela IV

RESUlTAdos DAS CNNS NA CLASSIFICAÇÃo MULTICLASSES COVID-19, Normal E PNEUMONIA VIRAL.

\begin{tabular}{|c|c|c|c|c|c|c|}
\hline & Acurácia & Sensibilidade & Especificidade & Precisão & Pontuação F1 & AUC \\
\hline VGG19 & $91,41 \%$ & $97,63 \%$ & $98,64 \%$ & $91,76 \%$ & $91,50 \%$ & $93,48 \%$ \\
VGG16 & $92,37 \%$ & $99,22 \%$ & $98,45 \%$ & $92,97 \%$ & $92,41 \%$ & $94,15 \%$ \\
InceptionV3 & $94,12 \%$ & $99,22 \%$ & $\mathbf{9 9 , 7 8 \%}$ & $94,54 \%$ & $94,12 \%$ & $95,47 \%$ \\
DenseNet201 & $\mathbf{9 4 , 5 2 \%}$ & $98,71 \%$ & $99,12 \%$ & $\mathbf{9 4 , 8 1 \%}$ & $\mathbf{9 4 , 5 5 \%}$ & $\mathbf{9 5 , 8 1 \%}$ \\
ResNet50 & $73,37 \%$ & $87,33 \%$ & $99,12 \%$ & $77,13 \%$ & $70,78 \%$ & $79,22 \%$ \\
Xception & $92,77 \%$ & $99,22 \%$ & $99,34 \%$ & $93,24 \%$ & $92,72 \%$ & $94,42 \%$ \\
InceptionResNetV2 & $93,32 \%$ & $98,71 \%$ & $99,10 \%$ & $93,56 \%$ & $93,39 \%$ & $94,94 \%$ \\
NASNetLarge & $94,28 \%$ & $\mathbf{9 9 , 4 9 \%}$ & $99,10 \%$ & $94,33 \%$ & $94,28 \%$ & $95,69 \%$ \\
\hline
\end{tabular}

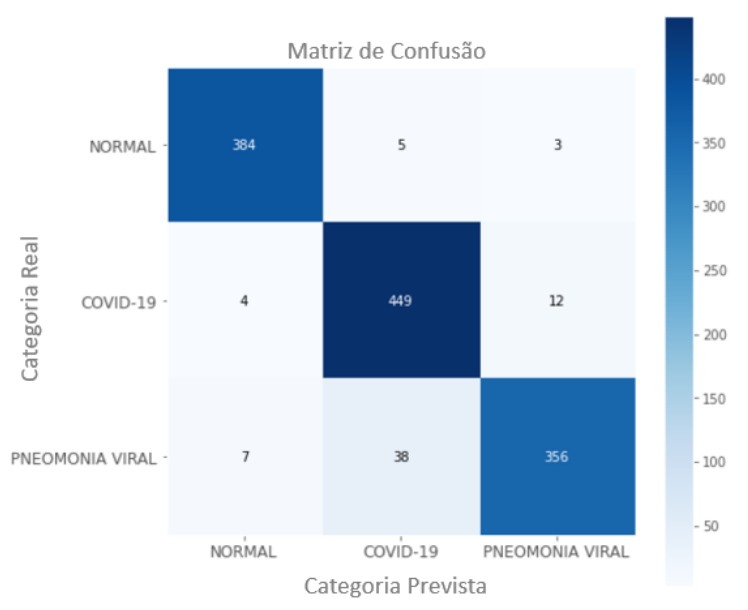

Figura 4. Matriz de confusão DenseNet2011 de COVID-19, Normal e Pneumonia Viral

maiores para a rede NASNetLarge, seguidos pela InceptionResNetV2, Xception, InceptioV3 e DenseNet201, no entanto, os resultados não seguiram essa ordem, variando dependendo do tipo de classificação, porém com variações pequenas.

Para avaliar melhor os resultados obtidos (Tabelas V e VI), foi realizado um comparativo com os melhores resultados obtidos com outros trabalhos que abordam como tema a classificação da COVID-19 em imagens de raio-x do tórax com base de dados distintas. Esse comparativo busca comparar os resultados alcançados na literatura com os resultados alcançados neste trabalho.

Na classificação binária entre COVID-19 e Normal, o modelo treinado que alcançou melhor desempenho obteve resultado abaixo apenas ao modelo DesNet201 [42] nas medidas de desempenho de acurácia e sensibilidade.

$\mathrm{Na}$ classificação multiclasse, a acurácia obtida foi inferior aos resultados dos trabalhos [42], [43], [45], [46] e [49], em contrapartida, a sensibilidade e especificidade foi superior as demais pesquisas, sendo essas as medidas de desempenho de maior importância para o diagnóstico na triagem utilizando imagens médicas.

\section{Conclus Ão}

Neste trabalho, foi realizada a aplicação de redes neurais convolucionais pre-treinadas (VGG19, VGG16, InceptionV3, ResNet50, DenseNet201, Xception, InceptionResNetV2 e NASNetLarge) em imagens de raio-X de tórax para três diferentes classes: COVID-19, Pneumonia Viral e Normal, 
Tabela V

COMPARATIVO ENTRE MODELOS DE CLASSIFICAÇÃO BINÁRIA.

\begin{tabular}{|c|c|c|c|c|}
\hline Trabalho & Tipo & Acurácia & Sensibilidade & Especificidade \\
\hline$[40]$ & COVID-Net - Binário & $93,3 \%$ & - & - \\
{$[13]$} & VGG19 - Binário & $90 \%$ & - & - \\
{$[41]$} & GDCNNN - Binário & $99 \%$ & - & - \\
{$[13]$} & DenseNet201 - Binário & $90 \%$ & - & - \\
{$[42]$} & DesNet201 - Binário & $99,70 \%$ & $99,70 \%$ & $99,55 \%$ \\
{$[43]$} & CoroNet-Binário & $95 \%$ & - & $97,5 \%$ \\
{$[44]$} & Modelos - Binário & $96,1 \%$ & - & - \\
Este trabalho & Xception & $\mathbf{9 9 , 6 4 \%}$ & $\mathbf{9 9 , 4 9 \%}$ & $\mathbf{9 9 , 7 7 \%}$ \\
\hline
\end{tabular}

Tabela VI

COMPARATIVO ENTRE MODELOS DE CLASSIFICAÇÃO MULTICLASSE.

\begin{tabular}{|c|c|c|c|c|}
\hline Trabalho & Tipo & Acurácia & Sensibilidade & Especificidade \\
\hline$[45]$ & VGG19 - Multiclasse & $95,67 \%$ & - & - \\
{$[45]$} & VGG16 - Multiclasse & $94,02 \%$ & - & - \\
{$[45]$} & InceptionV3 - Multiclasse & $93,56 \%$ & - & - \\
{$[46]$} & MobileNet-Multiclasse & $97,40 \%$ & $92,85 \%$ & $99,10 \%$ \\
{$[47]$} & InceptionV3 - Multiclasse & $93,4 \%$ & $92,3 \%$ & $88,8 \%$ \\
{$[42]$} & DesNet201 - Multiclasse & $97,94 \%$ & $97,95 \%$ & $98,80 \%$ \\
{$[48]$} & Método proposto - Multiclasse & $88,9 \%$ & $92,5 \%$ & $96,4 \%$ \\
{$[43]$} & CoroNet - Multiclasse & $99 \%$ & - & $98,6 \%$ \\
{$[40]$} & COVID-Net - Multiclasse & $93,3 \%$ & - & - \\
{$[49]$} & VGG16 - Multiclasse & $95,88 \%$ & - & - \\
{$[49]$} & VGG19-Multiclasse & $95,03 \%$ & - & - \\
Este trabalho & DenseNet201 & $\mathbf{9 4 , 5 2 \%}$ & $\mathbf{9 8 , 7 1 \%}$ & $\mathbf{9 9 , 1 2 \%}$ \\
\hline
\end{tabular}

com objetivo de classificar corretamente as imagens de raio$\mathrm{x}$ e identificar a COVID-19 para que possa ser utilizado no contexto de triagem medica para auxiliar no diagnostico. Para isso, foi considerando as medidas de desempenho de acurácia, sensibilidade, especificidade, precisão, pontuação F1 e AUC para a avaliar o desempenho dos modelos.

Durante os experimentos alguns modelos se destacaram, a Xception se destacou na classificação binária entre COVID19 e Normal, obtendo os melhores resultados na maioria das medidas de desempenho. Tendo em vista a triagem médica, tanto a Xception quanto a InceptionV3 e a DenseNet201 se provaram eficiente na classificação.

A DenseNet201, na classificação binária entre COVID-19 e Pneumonia Viral e na classificação multiclasse (entre as três classes), atingiu resultados superiores aos demais nas medidas de acurácia, precisão, pontuação F1 e AUC. Levando em conta a triagem, a classificação da Xception e a NASNetLarge alcançaram resultados melhores.

No comparativo entre outras pesquisas, a classificação binária entre COVID-19 e Normal, usando a Xception, obteve resultados bem próximos quando equiparado com a DenseNet201. Na classificação multiclasse, considerando a DenseNet201, o valor da acurácia foi inferior a alguns dos resultados obtidos pelos outros trabalhos, porém atingiu resultados melhores de sensibilidade e especificidade.

Alguns dos modelos atingiram bons resultados e são capazes de identificar nas imagens de raio-x a presença da COVID-19, contudo, se faz necessário de base de imagens maiores para ter um maior desempenho na classificação e confiabilidade no diagnostico. Outro ponto, são as imagens de Raio-X apresentaram alguns desafios, uma vez que possuem diferenças significativas entre elas, como o mal posicionamento da caixa torácica e ruídos de artefatos, o que dificulta o reconhecimento de padrões. Esses são fatores que implicam diretamente os resultados. Como trabalhos futuros serão utilizados fontes de dados maiores, exploração de outras arquiteturas de redes e técnicas de pré-processamento.

\section{AGRADECIMENTOS}

Os autores agradecem ao Programa de Pós Graduação de Engenharia Elétrica (PPGEE), ao Instituto Federal de Educação da Paraíba (IFPB), a CAPES e a FAPERJ pelo apoio no desenvolvimento deste trabalho.

\section{REFERÊNCIAS}

[1] A. E. Gorbalenya, S. C. Baker, R. Baric, R. J. d. Groot, C. Drosten, A. A. Gulyaeva, B. L. Haagmans, C. Lauber, A. M. Leontovich, B. W. Neuman, et al., "Severe acute respiratory syndrome-related coronavirus: The species and its viruses-a statement of the coronavirus study group," 2020.

[2] C. Huang, Y. Wang, X. Li, L. Ren, J. Zhao, Y. Hu, L. Zhang, G. Fan, J. Xu, X. Gu, et al., "Clinical features of patients infected with 2019 novel coronavirus in wuhan, china," The lancet, vol. 395, no. 10223, pp. 497-506, 2020.

[3] A. A. Elfiky, "Ribavirin, remdesivir, sofosbuvir, galidesivir, and tenofovir against sars-cov-2 rna dependent rna polymerase (rdrp): A molecular docking study," Life sciences, p. 117592, 2020.

[4] A. R. R. Freitas, N. M. d. Medeiros, L. C. V. Frutuoso, O. A. Beckedorff, L. M. A. d. Martin, M. M. d. M. Coelho, G. G. S. d. Freitas, D. R. Q. Lemos, and L. P. d. G. Cavalcanti, "Tracking excess deaths associated with the covid-19 epidemic as an epidemiological surveillance strategypreliminary results of the evaluation of six brazilian capitals," Revista da Sociedade Brasileira de Medicina Tropical, vol. 53, 2020.

[5] K. D. Toennies, "Advances in computer vision and pattern recognition," 2012.

[6] I. R. Silva, G. S. Silva, R. G. de Souza, W. P. dos Santos, and A. d. A. Roberta, "Model based on deep feature extraction for diagnosis of alzheimer's disease," in 2019 International Joint Conference on Neural Networks (IJCNN), pp. 1-7, IEEE, 2019. 
[7] R. Ranjbarzadeh, A. B. Kasgari, S. J. Ghoushchi, S. Anari, M. Naseri, and $\mathrm{M}$. Bendechache, "Brain tumor segmentation based on deep learning and an attention mechanism using mri multi-modalities brain images," Scientific Reports, vol. 11, no. 1, pp. 1-17, 2021.

[8] M. A. Al-Antari, S.-M. Han, and T.-S. Kim, "Evaluation of deep learning detection and classification towards computer-aided diagnosis of breast lesions in digital x-ray mammograms," Computer methods and programs in biomedicine, vol. 196, p. 105584, 2020.

[9] P. Lakhani and B. Sundaram, "Deep learning at chest radiography: automated classification of pulmonary tuberculosis by using convolutional neural networks," Radiology, vol. 284, no. 2, pp. 574-582, 2017.

[10] A. Gupta, S. Gupta, R. Katarya, et al., "Instacovnet-19: A deep learning classification model for the detection of covid-19 patients using chest X-ray," Applied Soft Computing, vol. 99, p. 106859, 2021.

[11] D. Riquelme and M. A. Akhloufi, "Deep learning for lung cancer nodules detection and classification in ct scans," AI, vol. 1, no. 1, pp. 28 67, 2020.

[12] E. Baratella, P. Crivelli, C. Marrocchio, A. M. Bozzato, A. D. Vito, G. Madeddu, L. Saderi, M. Confalonieri, L. Tenaglia, and M. A. Cova, "Severity of lung involvement on chest $\mathrm{x}$-rays in sars-coronavirus-2 infected patients as a possible tool to predict clinical progression: an observational retrospective analysis of the relationship between radiological, clinical, and laboratory data," Jornal Brasileiro de Pneumologia, vol. 46, no. 5, 2020.

[13] E. E.-D. Hemdan, M. A. Shouman, and M. E. Karar, "Covidx-net: A framework of deep learning classifiers to diagnose covid-19 in X-ray images," arXiv preprint arXiv:2003.11055, 2020.

[14] M. Nour, Z. Cömert, and K. Polat, "A novel medical diagnosis model for covid-19 infection detection based on deep features and bayesian optimization," Applied Soft Computing, vol. 97, p. 106580, 2020.

[15] I. U. Khan and N. Aslam, "A deep-learning-based framework for automated diagnosis of covid-19 using x-ray images," Information, vol. 11, no. 9, p. 419, 2020.

[16] C. B. Maior, J. M. Santana, I. D. Lins, and M. J. Moura, "Convolutional neural network model based on radiological images to support covid19 diagnosis: Evaluating database biases," Plos one, vol. 16, no. 3 , p. e0247839, 2021.

[17] H. Yu, L. T. Yang, Q. Zhang, D. Armstrong, and M. J. Deen, "Convolutional neural networks for medical image analysis: state-of-the-art, comparisons, improvement and perspectives," Neurocomputing, 2021.

[18] D. A. Rodrigues, "Deep learning e redes neurais convolucionais: reconhecimento automático de caracteres em placas de licenciamento automotivo," 2018.

[19] P. Singh, S. Chaudhury, and B. K. Panigrahi, "Hybrid mpso-cnn: Multilevel particle swarm optimized hyperparameters of convolutional neural network," Swarm and Evolutionary Computation, vol. 63, p. 100863, 2021.

[20] D. S. "Deep Acadamy, learning book." url=https://www.deeplearningbook.com.br/funcao-de-ativacao/. Accessed: 06-02-2021.

[21] DeepAI, "Funções de ativação: definição, características, e quando usar cada uma." https://iaexpert.academy/2020/05/25/funcoes-de-ativacaodefinicao-caracteristicas-e-quando-usar-cada-uma/. Accessed: 30-042021.

[22] I. Rodrigues, G. L. Santos, D. F. Sadok, and P. T. Endo, "Classifying covid-19 positive x-ray using deep learning models," IEEE Latin America Transactions, vol. 19, no. 6, pp. 884-892, 2021.

[23] M. J. Afridi, A. Ross, and E. M. Shapiro, "On automated source selection for transfer learning in convolutional neural networks," Pattern recognition, vol. 73, pp. 65-75, 2018.

[24] T. Chauhan, H. Palivela, and S. Tiwari, "Optimization and fine-tuning of densenet model for classification of covid-19 cases in medical imaging," International Journal of Information Management Data Insights, vol. 1, no. 2, p. 100020, 2021.

[25] K. Simonyan and A. Zisserman, "Very deep convolutional networks for large-scale image recognition," arXiv preprint arXiv:1409.1556, 2014.

[26] R. E. V. d. Silva, "Um estudo comparativo entre redes neurais convolucionais para a classificação de imagens," 2018.

[27] V. C. Rezende, M. Costa, A. Santos, and R. C. L. ao de Oliveira, "Aplicação de redes neurais convolucionais na classificação de doenças de plantas," in Anais do 14 Congresso Brasileiro de Inteligência Computacional (B. J. T. Fernandes and A. \{Pereira Júnior\}, eds.), (Curitiba, PR), pp. 1-7, ABRICOM, 2019.
[28] M. A. Zaki, S. Narejo, S. Zai, U. Zaki, Z. Altaf, and N. u Din, “Detection of ncov-19 from hybrid dataset of cxr images using deep convolutional neural network,"

[29] C. Szegedy, V. Vanhoucke, S. Ioffe, J. Shlens, and Z. Wojna, "Rethinking the inception architecture for computer vision," in Proceedings of the IEEE conference on computer vision and pattern recognition, pp. 2818 2826, 2016.

[30] G. Huang, Z. Liu, L. Van Der Maaten, and K. Q. Weinberger, "Densely connected convolutional networks," in Proceedings of the IEEE conference on computer vision and pattern recognition, pp. 4700-4708, 2017.

[31] F. Chollet, "Xception: Deep learning with depthwise separable convolutions," in Proceedings of the IEEE conference on computer vision and pattern recognition, pp. 1251-1258, 2017.

[32] S. Albahli and W. Albattah, "Deep transfer learning for covid-19 prediction: Case study for limited data problems," Current medical imaging, 2020.

[33] Z. Elhamraoui, "Inceptionresnetv2 simple introduction." url=https://medium.com/@ zahraelhamraoui1997/inceptionresnetv2simple-introduction-9a2000edcdb6/. Accessed: 27-05-2021.

[34] C. Szegedy, S. Ioffe, V. Vanhoucke, and A. Alemi, "Inception-v4, inception-resnet and the impact of residual connections on learning," in Proceedings of the AAAI Conference on Artificial Intelligence, vol. 31, 2017.

[35] M. W. H. Center, "Nasnetlarge." url=https://la.mathworks.com/. Accessed: 30-04-2021.

[36] Kaggle, "Covid-19 radiography database." url=https://www.kaggle.com/tawsifurrahman/covid19-radiographydatabase/. Accessed: 26-03-2021.

[37] C. Wang, D. Chen, L. Hao, X. Liu, Y. Zeng, J. Chen, and G. Zhang, "Pulmonary image classification based on inception-v3 transfer learning model," IEEE Access, vol. 7, pp. 146533-146541, 2019.

[38] D. Boyce, "Chapter 17 - evaluation of medical laboratory tests," in Orthopaedic Physical Therapy Secrets (Third Edition) (J. D. Placzek and D. A. Boyce, eds.), pp. 125-134, Elsevier, third edition ed., 2017.

[39] Tensorflow, "Keras applications." https://keras.io/api/applications/. Accessed: 03-06-2021.

[40] L. Wang, Z. Q. Lin, and A. Wong, "Covid-net: A tailored deep convolutional neural network design for detection of covid-19 cases from chest X-ray images," Scientific Reports, vol. 10, no. 1, pp. 1-12, 2020.

[41] R. Babukarthik, V. A. K. Adiga, G. Sambasivam, D. Chandramohan, and J. Amudhavel, "Prediction of covid-19 using genetic deep learning convolutional neural network (gdcnn)," IEEE Access, vol. 8, pp. 177647177666, 2020.

[42] M. E. Chowdhury, T. Rahman, A. Khandakar, R. Mazhar, M. A. Kadir, Z. B. Mahbub, K. R. Islam, M. S. Khan, A. Iqbal, N. Al Emadi, et al., "Can ai help in screening viral and covid-19 pneumonia?," IEEE Access, vol. 8, pp. 132665-132676, 2020.

[43] A. I. Khan, J. L. Shah, and M. M. Bhat, "Coronet: A deep neural network for detection and diagnosis of covid-19 from chest X-ray images,' Computer Methods and Programs in Biomedicine, vol. 196, p. 105581 2020.

[44] A. Narin, C. Kaya, and Z. Pamuk, "Automatic detection of coronavirus disease (covid-19) using X-ray images and deep convolutional neural networks," arXiv preprint arXiv:2003.10849, 2020.

[45] T. Tuncer, F. Ozyurt, S. Dogan, and A. Subasi, "A novel covid-19 and pneumonia classification method based on f-transform," Chemometrics and Intelligent Laboratory Systems, vol. 210, p. 104256, 2021.

[46] I. D. Apostolopoulos and T. A. Mpesiana, "Covid-19: automatic detection from X-ray images utilizing transfer learning with convolutional neural networks," Physical and Engineering Sciences in Medicine, vol. 43, no. 2, pp. 635-640, 2020.

[47] E. Benmalek, J. Elmhamdi, and A. Jilbab, "Comparing ct scan and chest X-ray imaging for covid-19 diagnosis," Biomedical Engineering Advances, p. 100003, 2021.

[48] Y. Oh, S. Park, and J. C. Ye, "Deep learning covid-19 features on cxr using limited training data sets," IEEE Transactions on Medical Imaging, vol. 39, no. 8, pp. 2688-2700, 2020.

[49] A. Makris, I. Kontopoulos, and K. Tserpes, "Covid-19 detection from chest X-ray images using deep learning and convolutional neural networks," in 11th Hellenic Conference on Artificial Intelligence, pp. 60 66, 2020. 